\title{
Taulinoplasty: the traction technique-a new extrathoracic repair for pectus excavatum
}

\author{
Carlos Bardaji ${ }^{1}$, Lluís Cassou ${ }^{2}$ \\ ${ }^{1}$ Department of Pediatric Surgery, Corporació Sanitària i Universitària ParcTaulí, Sabadell (Barcelona), Spain; ${ }^{2}$ Ventura Medical Technologies, La \\ Roca del Vallés (Barcelona), Spain \\ Correspondence to: Carlos Bardají, MD, PhD. Department of Pediatric Surgery, Corporació Sanitària i Universitària ParcTaulí, Av. ParcTaulí nº 1, \\ 08208 Sabadell (Barcelona), Spain. Email: cbardaji@tauli.cat.
}

Submitted Mar 08, 2016. Accepted for publication Sep 12, 2016.

doi: 10.21037/acs.2016.09.07

View this article at: http://dx.doi.org/10.21037/acs.2016.09.07

\section{Introduction}

The most frequent deformity of the ribcage is pectus excavatum (PE), which is described as the sternum sinking into the mediastinum. It is most likely caused by a congenital abnormality of the cartilage of the costosternal joints.

$\mathrm{PE}$ becomes apparent at the age of lactation, and in severe cases, can persist into adulthood. The associated symptoms depend on the severity of the deformity. Symptomatic patients with alterations in the complementary explorations are candidates for surgery.

Until the 1990s, the standard surgical treatment consisted of a subperichondral resection of the affected costosternal joints, with stabilization using an internal osteosynthesis of the fractures produced for the reduction of the defect.

In 1998, Donald Nuss published his non-invasive technique for lifting the sunken chest (1). Dr. Nuss' technique achieved the reduction of sternal collapse by rotating the pectus bar, based on the physical principle of levers which states that any weight can be lifted by a fulcrum and a long enough lever arm.

Open surgical procedures were shifted, with some exceptions only, by the introduction of the Nuss minimally invasive thoracoplasty (MIRPE), due to its excellent outcomes and the effectiveness of the bloodless reduction of the sternum achieved by levering from inside the chest.

Inspired by the same principles as in the Nuss Procedure, we thought that sternal lifting could also be possible through external traction, in order avoid invading the mediastinum or pleural cavity.
For this purpose, a plate for extrathoracic implantation and an associated traction system were designed. The plate has a specific shape and size to support and distribute the necessary loads over the defect. The traction system was designed to withstand the loads to be counteracted in the reduction of the sunken chest. The pectus plate and traction system are shown in detail in the video.

The forces required for the reduction of PE have been well studied by Fonkalsrud and Weber $(2,3)$. It seems clear that in adult patients with a Haller Index of 5 or more, the force needed to counteract the resistance of the sunken chest should be over $25 \mathrm{~kg}$.

For this reason, a series of in vitro tests on porcine sternums were made in order to determine the best configurations of screws according to the traction force required. The effect of a single standard $6.5 \mathrm{~mm}$ screw and a combination of two screws was calculated. Finally, a single $8 \mathrm{~mm}$ screw was constructed for evaluation. A MTS Bionics 858 high precision machine was used to measure the failure values (i.e., the force at which the traction of the screw breaks the bone). The results of the analysis are given in Newtons (mean and standard deviation).

(I) $6.5 \mathrm{~mm}$ screw $(390.8 \pm 82.9 \mathrm{~N})$;

(II) $2 \mathrm{~mm} \times 6.5 \mathrm{~mm}$ screw $(1011.9 \pm 115.7 \mathrm{~N})(\mathrm{P} \leq 0.01)$;

(III) $8 \mathrm{~mm}$ screw $(568.8 \pm 113.9 \mathrm{~N})(\mathrm{P} \leq 0.01)$.

The traction system consists of a screw and nut that are tightened progressively on the pectus plate until the reduction of the defect is achieved. The video shows the traction system reducing the sunken chest.

The aim of this article is to describe a non-invasive external traction system that reduces the sunken chest as 
additional aid in MIRPE, as well as an extrathoracic repair technique for PE.

\section{Surgical techniques (clinical case reports)}

\section{Traction technique as a complement in the Nuss technique (in an extreme Haller index case}

Clinical vignette. A 15-year-old boy presented with severe PE, kyphosis, lordosis, sternal rotation, herniation of the liver and exercise intolerance. Preoperative tests demonstrated a restrictive pattern on spirometry and Haller Index of 14.14 on the computed tomography (CT) scan.

Surgical technique:

(I) Preparation. The patient is placed in a in supine decubitus position with the arms in abduction. Orotracheal non-selective intubation and standard antibiotic prophylaxis is given. A $5 \mathrm{~mm}$ and $30^{\circ}$ thoracoscope, the traction system, the external platforms, the pectus bars, introducers and stabilizers should be prepared before the surgery;

(II) Exposure. Marking of key points. Reference points are marked on the deepest area of the sunken chest as well as on the desired entry and exit locations for the pectus bar and for the subcutaneous tunnels;

(III) Operation. Two subcutaneous tunnels are created at the desired level in relation to the defect. After drilling the hole, one double screw is placed at the deepest point of the sunken chest. The traction system is mounted over the external platform, coupled with the double screw and is progressively tightened. Under direct thoracoscopic view, the sternum is elevated gently and carefully. This maneuver reduces the Haller Index from 14.14 to less than 5. At this moment, the introducer is slid easily from one side of the chest to the other;

(IV) Completion. As a standard MIRPE, the pectus bar is rotated and both lateral stabilizers are fixed. The gas is evacuated by forced inspiration under water seal.

\section{Taulinoplasty: fastening with screws}

Clinical vignette. A 6-year-old boy presented with $\mathrm{PE}$, lordosis, moderate sternal rotation, pollen allergy and exercise intolerance. The complementary tests demonstrated a restrictive pattern on spirometry and Haller
Index of 4.31 on the CT scan.

(I) Preparation. The patient is placed in a supine decubitus position with arms in abduction. Orotracheal non-selective intubation and standard antibiotic prophylaxis is given. The traction system, pectus plates, screws and tools should be prepared before the surgery;

(II) Exposure. A 3-4 cm long transverse incision is made at the deepest point of the sunken chest. A pouch is dissected to accommodate the pectus plate at its desired position. The dissection should be longer on one of the two sides of the chest in order to allow the insertion of the plate. The two side wings of the plate should rest on the two zenithal points of both hemithorax;

(III) Operation. After drilling the hole, one double screw is placed exactly at the deepest point of the sunken chest. Then, the pectus plate is housed on the pouch and the traction system is mounted and coupled with the double screw. By tightening the traction system, the defect is progressively reduced. The plate is fixed by screws to the chest bone and the traction system is easily removed. A flat screw is placed to cover the remaining hole;

(IV) Completion. The wound is closed with absorbable sutures and subcuticular non-reabsorbable running sutures.

\section{Taulinoplasty: fixation with steel wires}

Clinical vignette. A 13-year-old girl presented with slow growth development treated with Growth Hormone, PE with a symmetric defect, lordosis, and exercise intolerance. The complementary tests demonstrated a restrictive pattern on spirometry and Haller Index of 5.26 on the CT scan.

(I) Preparation. Same as in procedure \#2;

(II) Exposure. Same as in procedure \#2;

(III) Operation. Same as in procedure \#2. At least six steel wires are placed: two through the sternum and two on each side through the best placed ribs. Then, the pectus plate is housed on the pouch and the traction system is mounted and coupled with the double screw. By tightening the traction system, the defect is progressively reduced. The plate is then fixed by locking all of the steel wires. Finally, one more steel wire is passed through the xiphoid appendix and the lowest hole of the plate.

(IV) Completion. Same as in procedure \#2. 


\section{Taulinoplasty: fixation with peek (Zip Fix)}

Clinical vignette. A 15 -year-old girl presented with symmetric $\mathrm{PE}$ and exercise intolerance. The complementary tests demonstrated a restrictive pattern on spirometry and Haller Index of 4.10 on the CT scan.

(I) Preparation. Same as in procedure \#2;

(II) Exposure. Same as in procedure \#2;

(III) Operation. Same as in procedure \#2. With the help of a rib dissector, two Zip Fix ribbons are placed around the best placed ribs of each side. Then, the pectus plate is housed on the pouch and the traction system is mounted and coupled with the double screw. By tightening the traction system, the defect is progressively reduced. The plate is fixed by tensing the Zip Fix with a special tool;

(IV) Completion. Same as in procedure \#2.

\section{Comments}

\section{Clinical results}

The Taulinoplasty with traction technique has been performed in 18 cases (15 boys and 3 girls) ranging from 6 to 24.3 years (mean 14.7 years). ( $\bar{x}=$ arithmetic mean). The Haller Index before the surgery was 3.51-13.23 (mean 5.16). The average duration of the intervention was 45 minutes. In all the cases, the intervention could be performed after the initial planning and reduction of the defect was achieved. Some minor complications were experienced. In one of the first cases, a small seroma appeared which resolved without the need for a drain. In two older patients (17 and 19 years old), moderate loosening of the plate was observed after 6 and 7 weeks respectively after operation. Surgical retightening was successfully performed. The follow up ranges from 18 to 32 months. The Haller Index after surgery in all cases demonstrated normal values (mean 3.19). To date, five plates have been removed following the same principles of the MIRPE bar removals following successful procedures. During the surgical removal process, no difficulties arose and the scar was shortened and improved.

\section{Advantages}

The preliminary results of this study are encouraging but obviously, the series is still short and it is necessary to extend the trial period. The main advantage demonstrated with this approach is avoiding invasion of the mediastinum and the pleural cavity in order to reduce the main complications of MIRPE and the risk of injury to vital organs $(4,5)$. The aim of this technique is to offer another therapeutic option within the surgical arsenal, and in particular to patients who are fearful of being operated on by other procedures. Due to this fear, despite the congenital nature of the condition, patients are being operated on too late and therefore increasing the progression of the defect and surgical difficulties.

\section{Caveats}

Special attention should be paid to the placement of the traction system. The screw must be placed in the exact spot, avoiding the neighboring area to the xiphoid. The drilling of the sternum must be performed at 90 degrees to the plane of the operating table and not in relation to the plane of the sternum, to prevent breaking the bone. If this should happen, a secondary hole next to the above can be made without any issues. The hole can be created manually or using the electric drill. With regards to the fasteners, we believe that in young children screws can be used, but in pubertal children or adults, it is best to use steel wire, Zip Fix or both. As a limitation of the study, very asymmetrical or long funnel (Grand Canyon) chested patients should not be considered candidates for this procedure. Potential for improvement therefore lies in further developing the procedure to include the treatment of such deformities, as well as cases where other surgical techniques have been unsuccessful. According to this experience, the learning curve is shorter than with other PE surgical repair techniques.

\section{Acknowledgements}

Funding: This work was partially funded with a grant nr. VALTEC 08-2-0045 from the Generalitat de Catalunya.

\section{Footnote}

Conflicts of Interest: The authors have no conflicts of interest to declare.

\section{References}

1. Nuss D, Kelly RE Jr, Croitoru DP, et al. A 10-year review of a minimally invasive technique for the correction of pectus excavatum. J Pediatr Surg 1998;33:545-52.

2. Fonkalsrud EW, Reemtsen B. Force required to elevate 
the sternum of pectus excavatum patients. J Am Coll Surg 2002;195:575-7.

3. Weber PG, Huemmer HP, Reingruber B. Forces to be overcome in correction of pectus excavatum. J Thorac Cardiovasc Surg 2006;132:1369-73.

4. Kelly RE Jr, Mellins RB, Shamberger RC, et al. Multicenter study of pectus excavatum, final report:

Cite this article as: Bardají C, Cassou L. Taulinoplasty: the traction technique-a new extrathoracic repair for pectus excavatum. Ann Cardiothorac Surg 2016;5(5):519-522. doi: 10.21037/acs.2016.09.07 complications, static/exercise pulmonary function, and anatomic outcomes. J Am Coll Surg 2013;217:1080-9.

5. Schaarschmidt K, Lempe M, Schlesinger F, et al. Lessons learned from lethal cardiac injury by nuss repair of pectus excavatum in a 16-year-old. Ann Thorac Surg 2013;95:1793-5. 